\title{
Eficiência e resposta à aplicação de nitrogênio de genótipos de feijão comum cultivados em várzea tropical do Estado do Tocantins
}

\section{Efficiency and response to nitrogen application of common bean genotypes grown in lowland tropical rainforest in the state of Tocantins}

\author{
Sérgio Alves de Sousa ${ }^{1}$, Joedna Silva ${ }^{1}$, Danilo Pereira Ramos ${ }^{1}$, Taynar Coelho de Oliveira, \\ Luiz Antonio de Menezes Gonzaga ${ }^{1}$ e Rodrigo Ribeiro Fidelis ${ }^{1 *}$
}

${ }^{1}$ Departamento de Agronomia; Universidade Federal do Tocantins; 77402-970; Gurupi - TO - Brasil.

\begin{abstract}
The common bean is a crop with big socioeconomic interest in many countries, being a crop with high nutrient demand, mainly nitrogen which is uptaken in big amounts. Thus, it is necessary the adoption of cultivars which are efficient and responsive to nitrogen use. So, this study was carried out in order to evaluate the nitrogen use efficiency and response of common bean genotypes grown in irrigated lowland soil at State of Tocantins. The study was carried out in two trials (high and low nitrogen level) in a completely randomized blocks design, being evaluated 25 genotypes in three replications. To simulate environments with low and high $N$ level, doses of 0 and $120 \mathrm{~kg} \mathrm{ha}^{-1}$ of $\mathrm{N}$ were utilized respectively. The genotypes were sorted about nitrogen use efficiency though their grain yield. There is difference about nitrogen use efficiency and response to nitrogen application among the evaluated genotypes.
\end{abstract}

Key-words: Phaseolus vulgaris, nutritional stress, genotypes evaluation

\section{INTRODUÇÃO}

O feijão comum (Phaseolus vulgaris L.) é a espécie de maior interesse econômico dentro do gênero Phaseolus, sendo de grande importância social por ser integrante fundamental da dieta de muitos países devido seu alto valor nutritivo (Santos e Gavilanes, 2006; Binotti et al., 2009; Posse et al., 2010).

O Brasil figura entre os maiores produtores de feijão do mundo sendo estimada para a safra 2011/2012 uma produção de 3,5 milhões de toneladas numa área plantada de 3,8 milhões de hectares com produtividade média de $907 \mathrm{~kg} \cdot \mathrm{ha}^{-1}$. Neste contexto, para o Estado do Tocantins, as estimativas são de 43,8 mil hectares plantados, com produção de 69 mil toneladas e produtividade de $1.576 \mathrm{~kg} . h a^{-1}$ (Conab, 2012). Embora sejam médias baixas, em ensaios experimentais já são observados rendimentos superiores aos 3.000 kg.ha ${ }^{-1}$ (Lemos et al., 2004; Mechi et al., 2005; Salgado et al., 2011), demostrando assim o grande potencial do Estado no cultivo desta cultura. Este aumento no rendimento deu-se também com a introdução do feijoeiro em áreas de várzeas tropicais no período da entressafra, onde se alcança ainda a redução dos custos e rendimentos significativos (Aidar e Kuthcouski, 2008).

Dentre vários fatores considerados problemáticos ao cultivo do feijoeiro em áreas de várzea, a adubação nitrogenada merece destaque pelo fato do nitrogênio ser o nutriente absorvido em maior quantidade pela cultura do feijão, a demanda ser maior em várzeas do que nos cultivos em terras altas e assim sua carência ser bastante limitante a

*Author for correspondence: fidelisrr@uft.edu.br

J. Biotec. Biodivers. v. 3, N.3: pp. 31-37, Aug. 2012 
obtenção de altos rendimentos (Santos e Fageria, 2007; Fageria et al., 1996; Sant'Ana et al., 2010; Posse et al., 2010).

$\mathrm{O}$ uso inadequado da adubação nitrogenada tornou-se preocupante devido acarretar redução nos rendimentos, aumentos nos custos de produção e principalmente ocasionar poluição ambiental com as perdas que podem ocorrer por volatilização, lixiviação e desnitrificação (Santos e Fageria, 2007, Sant'Ana et al., 2010). Diante desta preocupação, tem-se buscado soluções para maximizar o uso do nitrogênio, dentre elas a utilização de cultivares que sejam mais eficientes no uso de $\mathrm{N}$ e que também respondam de forma satisfatória a adubação nitrogenada, promovendo ganhos de produtividade e redução de custos, principalmente em solos deficientes de nitrogênio. Vários são os estudos com relatos do efeito da adubação nitrogenada em diversas culturas como milho (Fidelis et al., 2010), arroz (Cancellier et al., 2011; Kischel et al., 2011) e feijão (Santos et al., 2003; Salgado et al., 2011), assim como o agrupamento dos genótipos de acordo com a capacidade destes de resposta e utilização do nitrogênio. Porém, estudos com avaliação da eficiência e resposta ao uso do nitrogênio na cultura do feijão em condições de várzea tropical ainda são bastante incipientes.

Diante deste contexto, objetivou-se com este estudo avaliar a eficiência e resposta ao uso de nitrogênio de genótipos de feijão comum cultivados em solos de várzea tropical do estado do Tocantins.

\section{MATERIAL E MÉTODOS}

Os experimentos (alto e baixo nível de nitrogênio) foram conduzidos no município de Formoso do Araguaia-TO, em solo tipo Gleissolo (Embrapa, 2006), de várzea irrigada do módulo I-14 da COPERJAVA (Cooperativa Mista do Vale do Araguaia), nas coordenadas geográficas $11^{\circ} 48^{\prime} \mathrm{S}$ e $49^{\circ} 37^{\prime} \mathrm{W}$, a $227 \mathrm{~m}$ de altitude.

Anterior a implantação do experimento foram coletadas amostras de solo na camada de $0-20 \mathrm{~cm}$ de profundidade para análise química e física do solo, obtendo os seguintes resultados: $\mathrm{pH}$ em $\mathrm{H}_{2} \mathrm{O}$ $=5,44 ; \mathrm{M} . \mathrm{O}\left(\mathrm{g} \mathrm{dm}^{-3}\right)=71,71 ; \mathrm{P}\left(\right.$ Melich $\left.^{-1}\right)=9,61$ $\mathrm{mg} \mathrm{dm}{ }^{-3} ; \mathrm{Ca}=3,07 \mathrm{cmol}_{\mathrm{c}} \mathrm{dm}^{-3} ; \mathrm{Mg}=1,12 \mathrm{cmol}_{\mathrm{c}}$ $\mathrm{dm}^{-3} ; \mathrm{H}+\mathrm{Al}=4,36 \mathrm{cmol}_{\mathrm{c}} \mathrm{dm}^{-3} ; \mathrm{K}=0,03 \mathrm{cmol}_{\mathrm{c}} \mathrm{dm}^{-}$ 3; $\mathrm{SB}=4,22 \mathrm{cmol}_{\mathrm{c}} \mathrm{dm}^{-3} ; 547,35 \mathrm{~g} \mathrm{~kg}^{-1}$ de areia; $166,7 \mathrm{~g} \mathrm{~kg}^{-1}$ de sílte e $285,99 \mathrm{~g} \mathrm{~kg}^{-1}$ de argila.

$\mathrm{O}$ delineamento experimental utilizado em ambos os experimentos foi o de blocos ao acaso com três repetições. Cada unidade experimental foi constituída por quatro linhas de $5,0 \mathrm{~m}$ de comprimento, espaçadas de 0,45 m. Como área útil foram utilizadas as duas linhas centrais desprezando-se as duas linhas laterais e $0,5 \mathrm{~m}$ das extremidades das duas linhas centrais, ficando com linhas de 4,0 m de comprimento e área útil de 3,6 $\mathrm{m}^{2}$.

Foram utilizadas quatro linhagens (CNFC-10406, CNFC-10467, CNFC-10470 e VC-3) e vinte e um genótipos (BRS-Aporé, BRS-Esplendor, BRSGrafite, BRS-Horizonte, IAC-Alvorada, IACDiplomata, IAC-Eté, IAC-Galante, IAC-Una, IPR139, IPR-Chopim, IPR-Colibri, IPR-Corujinha, IPR-Gralha, IPR-Saracura, IPR-Tiziu, BRSMarfim, BRS-Majestoso, Uirapuru e Safira).

O preparo do solo foi realizado de forma convencional, ou seja, com uma aração e duas gradagens. A semeadura foi realizada no dia 4 de junho de 2011. O plantio foi realizado visando obter estande final de 12 plantas por metro linear. A adubação de semeadura foi realizada no sulco de plantio com base nos resultados da análise química e física do solo, sendo aplicados 500 $\mathrm{kg} \cdot \mathrm{ha}^{-1}$ do adubo formulado $0-16-16+6 \% \mathrm{~S}+$ micro. A adubação de cobertura no experimento de alto nível de nitrogênio foi realizada aplicando-se 120 $\mathrm{kg} \mathrm{ha}{ }^{-1}$, aos 20 dias após a emergência das plântulas, na forma de sulfato de amônia. (Rosolem e Marubayashi, 1994).

No experimento de baixo nível do nutriente não foi realizada adubação nitrogenada, pois considerando uma média de $5 \%$ de mineralização do N-orgânico por ano (Camargo et al., 2008), encontrava-se com níveis adequados para simulação dessa condição ( $\left.35 \mathrm{~kg} \cdot \mathrm{ha}^{-1} \mathrm{de} \mathrm{N}\right)$.

Foi realizado o tratamento de sementes com aplicação de $300 \mathrm{ml} / 100 \mathrm{~kg}$ de sementes do fungicida pertencente ao grupo químico benzimidazol.

Durante a condução do experimento para o controle das plantas daninhas (Ciperáceas) foram aplicados 1,2 L.ha ${ }^{-1}$ de um herbicida pertencente ao grupo químico benzotiadiazinonan. Para o controle dos insetos (Diabrotica speciosa) foi realizada a aplicação de $0,6 \mathrm{~L} \cdot \mathrm{ha}^{-1}$ de um inseticida pertencente ao grupo químico organofosforado e para o controle de doenças (Sclerotinia sclerotiorum) foi realizado uma aplicação de 70 gramas do produto comercial de um fungicida pertencente ao grupo químico benzimidazol.

A avaliação de produtividade foi realizada colhendo os grãos de toda a área útil da parcela e 
depois através da massa com correção da umidade para $13 \%$ foi estimada a produtividade em $\mathrm{kg} \mathrm{ha}^{-1}$. Para diferenciação dos genótipos, foi utilizada a metodologia proposta por Fageria e Kluthcouski (1980), que sugerem a classificação das cultivares quanto à eficiência no uso e resposta a aplicação do nitrogênio (eficiência e resposta - ER). Onde a utilização do nutriente é definida pela média de produtividade de grãos em baixo nível. A resposta à utilização do nutriente é obtida pela diferença entre a produtividade de grãos nos dois níveis dividida pela diferença entre as doses utilizando a seguinte fórmula:

Onde:

$$
\alpha_{N}=(\mathrm{PNN}-\mathrm{PBN}) / \mathrm{DEN},
$$

$\alpha_{\mathrm{N}}=$ Índice de resposta;

PNN = Produção com nível ideal de nutriente;

$\mathrm{PBN}=$ Produção com baixo nível do nutriente e;

DEN $=$ Diferença entre as doses aplicadas $\left(\mathrm{kg} \cdot \mathrm{ha}^{-1}\right)$.

Foi utilizada uma representação gráfica no plano cartesiano para classificar os genótipos. No eixo das abscissas (x), encontra-se a eficiência na utilização do nitrogênio e no eixo das ordenadas (y), a resposta à sua utilização. O ponto de origem dos eixos é a eficiência média e a resposta média das cultivares. No primeiro quadrante são

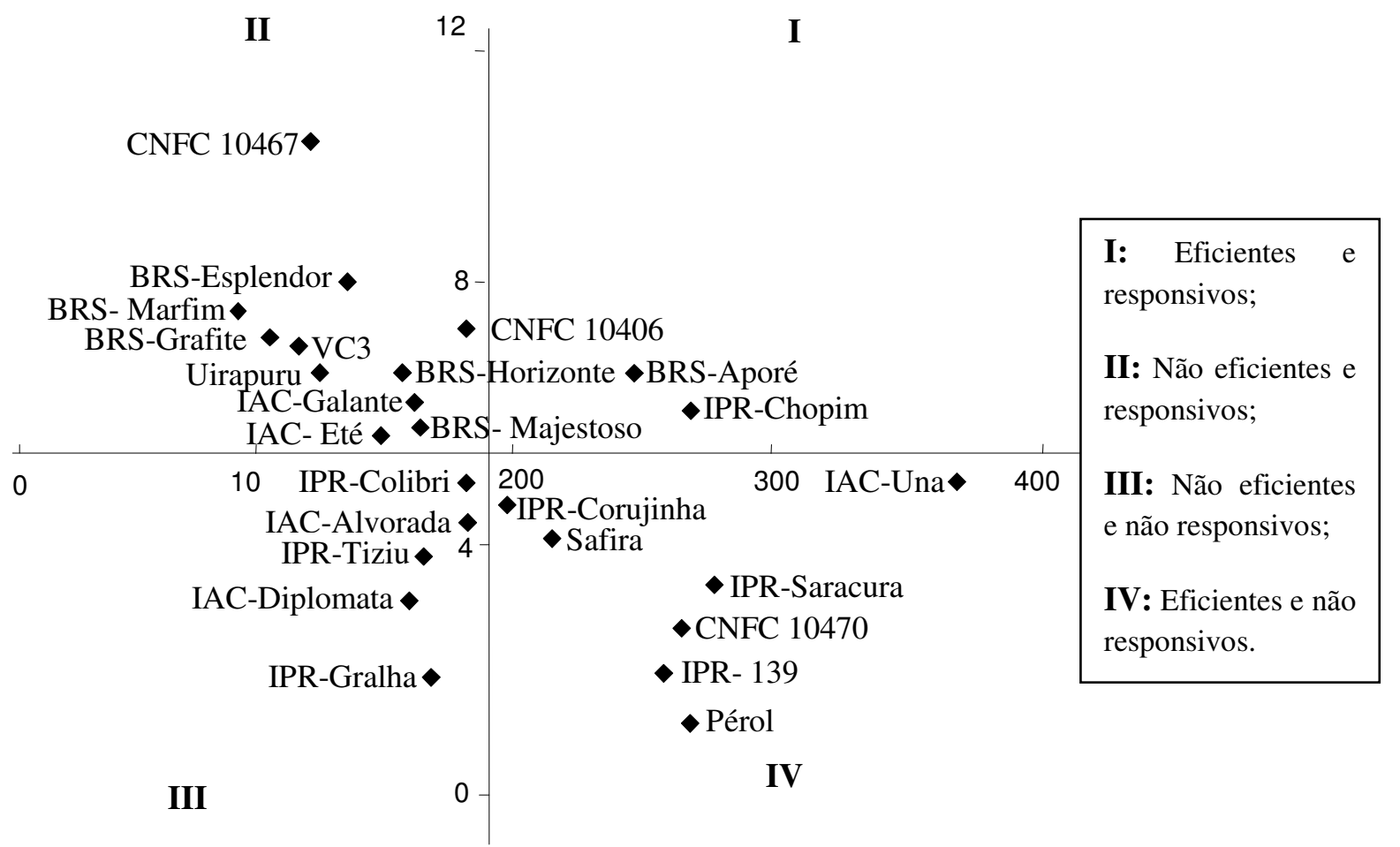

Figura 1. Eficiência no uso e resposta a aplicação de nitrogênio em genótipos de feijão, segundo metodologia proposta por Fageria e Kluthcouski (1980). representados os genótipos eficientes e responsivos; no segundo, os não eficientes e responsivos; no terceiro, os não eficientes e não responsivos e no quarto, os eficientes e não responsivos.

\section{RESULTADOS E DISCUSSÃO}

Pela metodologia adotada para diferenciação dos genótipos, a classificação quanto à eficiência e resposta ao uso do $\mathrm{N}$ é apresentada na Figura 1, onde $44 \%$ dos 25 genótipos foram classificados como não eficientes e responsivos, $28 \%$ como eficientes e não responsivos, $20 \%$ não eficientes e não responsivos e $8 \%$ eficientes e responsivos. Observa-se que os genótipos apresentaram comportamento diferenciado quanto à eficiência no uso do nitrogênio (Figura 1). Esta diversidade na eficiência de uso do $\mathrm{N}$ entre os genótipos de feijão também foi observada por Santos e Fageria (2007) em avaliação de cultivares de feijão quanto a eficiência e resposta ao nitrogênio, em função do manejo da adubação nitrogenada em condições de várzea. $\mathrm{O}$ que também pôde ser confirmado por Fageria (1998), porém, em estudo avaliando a eficiência de genótipos de feijão quanto à utilização de P. 
Foram identificados nove genótipos (36\%) como eficientes à utilização do nitrogênio, pois em condições de baixo nível de $\mathrm{N}$ apresentaram rendimentos superiores a média geral, sendo representados no primeiro e quarto quadrantes da Figura 1. Para Rotili et al. (2010) esta eficiência pode ser atribuída ao fato de esses genótipos serem mais eficientes nos processos metabólicos que envolvem desde a absorção do nutriente até a sua redistribuição dentro da planta. Epstein e Bloom (2004) também afirmam que a eficiência no uso do nutriente é resultado da soma da eficiência de utilização com a eficiência de absorção. Estes genótipos classificados como eficientes são indicados para cultivos em que grandes doses de adubação nitrogenada não são utilizadas e os produtores são limitados a baixo nível tecnológico (Cancellier et al., 2011).

Quanto à resposta a aplicação da adubação nitrogenada, houve equilíbrio entre os genótipos avaliados, sendo classificados 13 (52\%) como responsivos. Na representação gráfica (Figura 1), os genótipos responsivos se encontram nos dois primeiros quadrantes. Para Salgado (2011) estes genótipos são de interesse, pois quando cultivados em ambientes adequados de adubação, respondem satisfatoriamente aos incrementos na dose do nutriente.

Somente dois genótipos (8\%) foram classificados como eficientes e responsivos, sendo eles BRSAporé e IPR-Chopim (primeiro quadrante da Figura 1) que além de obterem rendimento acima da média geral em condições de baixo nitrogênio também obtiveram boa resposta ao acréscimo da adubação nitrogenada, com índices de resposta de 5,54 e 5,51 , respectivamente (Tabela 1 ). Segundo Salgado (2011) e Cancellier et al. (2011) os genótipos representados neste primeiro quadrante expressam uma possível adaptação tanto a condições de déficit de nitrogênio como a condições de ótima disponibilidade, e de acordo com Rotilli et al. (2010), estes genótipos são recomendados para cultivos agrícolas que adotam desde baixo a alto nível tecnológico.

No segundo quadrante estão representados os 11 genótipos (44\%) considerados não eficientes e responsivos, pois embora tenham apresentado resposta a adubação nitrogenada obtendo bom rendimento em condições adequadas de nitrogênio, em ambiente de deficiência de $\mathrm{N}$ não alcançaram o rendimento médio. Estes genótipos são indicados para produtores que dispõem de alta tecnologia, devido ao alto potencial de resposta a adubação nitrogenada (Rotilli et al., 2010; Salgado, 2011).

No terceiro quadrante encontram-se cinco genótipos que foram classificados como não eficientes e não responsivos, representando $20 \%$ dos genótipos avaliados, sendo eles, IPR-Colibri, IAC-Alvorada, IPR-Tiziu, IAC-Diplomata e IPRGralha. Estes genótipos apresentaram rendimentos abaixo da média em ambos os ambientes estudados não sendo indicados desta forma para cultivos agrícolas (Rotilli et al., 2010; Salgado, 2011).

Os genótipos IAC-Una, IPR-Corujinha, Safira, IPR-Saracura, CNFC-10470, IPR-139 e Pérola foram os genótipos classificados no quarto quadrante como eficientes e não responsivos. Estes genótipos são assim classificados devido terem produzido acima da média no ambiente de baixo nitrogênio e quando submetidos à dose elevada não responderam satisfatoriamente com rendimentos abaixo da média. O genótipo IACUna apresentou rendimento em condições de alto nitrogênio acima da média, porém seu índice de resposta $(4,93)$ ficou abaixo da média geral $(5,25)$. de índice de resposta (Tabela 1).

Na Tabela 1 são apresentados, a eficiência no uso de $\mathrm{N}$ que é o rendimento em condições de baixo nitrogênio, o índice de resposta obtido pela metodologia descrita por Fageria e Kluthcouski (1980) e a diferença de produtividade entre os ambientes (alto e baixo).

$\mathrm{O}$ genótipo IAC-Una apresentou a maior produtividade em condições de baixo nitrogênio com 365,61 kg.ha ${ }^{-1}$ (Tabela 1), confirmando sua eficiência e possível rusticidade.

Os índices de resposta variaram de 1,32 a 10,07. Esta variação é inferior a encontrada por Salgado (2011) em estudo semelhante, porém em terras altas $(-9,9$ a 16,1$)$ e também inferior a encontrada por Santos e Fageria (2007) que foi de 2 a 18 para adubação nitrogenada aplicada a lanço e em condições de várzea. O genótipo CNFC-10467 apresentou o maior índice de resposta com 10,07, o que significa dizer que para cada quilo de nitrogênio aplicado no solo houve um acréscimo de $10,07 \mathrm{~kg}$ de grãos, mostrando assim a grande capacidade de resposta desta linhagem quando submetido a condições de alta dose de N. Este índice está abaixo do maior valor encontrado por Salgado (2011) que foi de 16,1. Este índice de resposta refletiu diretamente nos incrementos de produtividade, onde o genótipo CNFC-10467 
atingiu $976 \%$ de aumento com a adição do adubo nitrogenado (Tabela 1).

Todos os genótipos considerados responsivos obtiveram índices de resposta acima de 5,25, com incrementos de produtividade variando de 57 a $1.049 \%$ (Tabela 1).

Tabela 1. Médias de eficiência agrônomica (EA), índice de resposta (IR) e diferença de produtividade de grãos (DPG) de 25 genótipos cultivados em área de várzea Tropical do Estado do Tocantins, na entressafra de 2011.

\begin{tabular}{|c|c|c|c|c|}
\hline Genótipos & EA $\left({\left.\mathrm{kg} . h a^{-1}\right)}^{-1}\right.$ & IR & DPG (kg.ha $\left.{ }^{-1}\right)$ & DPG (\%) \\
\hline CNFC 10467 & $123,74 \mathrm{a}$ & 10,07 & 1208,84 & 977 \\
\hline IAC Una & $365,61 \mathrm{a}$ & 4,93 & 591,78 & 162 \\
\hline CNFC 10406 & $192,63 \mathrm{a}$ & 7,07 & 848,07 & 440 \\
\hline BRS Esplendor & $136,49 \mathrm{a}$ & 7,82 & 937,93 & 687 \\
\hline IPR Chopim & $265,24 \mathrm{a}$ & 5,51 & 661,12 & 250 \\
\hline BRS Aporé & $240,14 \mathrm{a}$ & 5,54 & 664,73 & 277 \\
\hline BRS Horizonte & $172,13 \mathrm{a}$ & 6,55 & 785,89 & 457 \\
\hline IAC Galante & $186,67 \mathrm{a}$ & 6,08 & 729,41 & 391 \\
\hline BRS Majestoso & $183,91 \mathrm{a}$ & 5,70 & 684,21 & 372 \\
\hline BRS Grafite & $102,74 \mathrm{a}$ & 7,03 & 844,15 & 822 \\
\hline VC3 & $112,65 \mathrm{a}$ & 6,86 & 823,62 & 731 \\
\hline Uirapuru & $126,46 \mathrm{a}$ & 6,58 & 790,17 & 625 \\
\hline BRS Marfim & $82,59 \mathrm{a}$ & 7,22 & 866,74 & 1049 \\
\hline IPR Colibri & $192,79 \mathrm{a}$ & 5,17 & 620,95 & 322 \\
\hline IPR Saracura & $277,00 \mathrm{a}$ & 3,59 & 431,20 & 156 \\
\hline IAC Eté & $150,30 \mathrm{a}$ & 5,41 & 649,11 & 432 \\
\hline Safira & $207,05 \mathrm{a}$ & 4,31 & 516,98 & 250 \\
\hline IPR Corujinha & $198,93 \mathrm{a}$ & 4,35 & 522,42 & 263 \\
\hline CNFC 10470 & $253,28 \mathrm{a}$ & 3,28 & 393,54 & 155 \\
\hline IAC Alvorada & $192,25 \mathrm{a}$ & 4,29 & 514,75 & 268 \\
\hline IPR Tiziu & $171,69 \mathrm{a}$ & 4,19 & 502,53 & 293 \\
\hline IPR 139 & $270,58 \mathrm{a}$ & 2,12 & 254,06 & 94 \\
\hline IAC Diplomata & $166,83 \mathrm{a}$ & 3,76 & 450,62 & 270 \\
\hline Pérola & $279,05 \mathrm{a}$ & 1,32 & 158,82 & 57 \\
\hline IPR Gralha & $171,26 \mathrm{a}$ & 2,40 & 287,58 & 168 \\
\hline M̀iédias & 192,88 & 5,25 & & \\
\hline
\end{tabular}

Médias seguidas de mesma letra minúscula na coluna não diferem entre si pelo teste Scott-Knott a 5\% de probabilidade.

\section{CONCLUSÕES}

Existe diferença quanto à eficiência e reposta à aplicação de nitrogênio entre os genótipos avaliados.

Os genótipos BRS-Aporé e IPR-Chopim são indicados para cultivo agrícola em áreas de várzea no estado do Tocantins por serem eficientes e responsivos ao uso do nitrogênio.

Os genótipos IPR-Colibri, IAC-Alvorada, IPRTiziu, IAC-Diplomata e IPR-Gralha não são recomendados para cultivos em várzea no estado do Tocantins.

\section{RESUMO}

O feijão comum trata-se de uma cultura de grande interesse sócio-econômico em vários países, sendo muito exigente em nutrientes, principalmente $\mathrm{o}$ nitrogênio que é absorvido em grandes quantidades. Desta forma, faz-se necessário a adoção de cultivares que sejam eficientes e responsivos ao uso de nitrogênio. Objetivou-se com este estudo avaliar a eficiência e resposta ao uso de nitrogênio de genótipos de feijão comum cultivados em solos de várzea irrigada do estado do Tocantins. $\mathrm{O}$ estudo foi conduzido em dois experimentos (alto e baixo nível de nitrogênio) com delineamento em blocos casualizados, sendo avaliados 25 genótipos, em três repetições. Para a simulação dos ambientes de baixo e alto nível de $\mathrm{N}$ foram utilizadas as doses de 0 e $120 \mathrm{~kg} \mathrm{ha}^{-1}$ de $\mathrm{N}$, respectivamente. Através 
da produtividade de grãos os genótipos foram classificados quanto à eficiência e resposta a aplicação de nitrogênio. Existe diferença quanto à eficiência e resposta a aplicação de nitrogênio entre os genótipos avaliados.

Palavras-chave: Phaseolus vulgaris, estresse nutricional, avaliação de genótipos

\section{REFERÊNCIAS}

Aidar, H.; KluthcouskI, J. (2008), Relatório de Atividades de Pesquisa com o Feijoeiro Comum nas Várzeas Tropicais do Tocantins: Adequações Fitotécnicas com o Feijoeiro Cultivado em Sucessão ao Arroz Irrigado em Várzeas Tropicais. Santo Antônio de Goiás: Embrapa, (Documentos), 225, 40p.

Binotti, F. F. S.; Arf, O.; Sá, M. E.; Buzetti, S.; Alvarez, A. C. C.; Kamimura, K. M. (2009), Fontes, doses e modo de aplicação de nitrogênio em feijoeiro no sistema plantio direto. Bragantia, 68, 473-481.

Camargo, F. A. O.; Silva, L. S.; Gianello, C.; Tedesco, M. J. Nitrogênio orgânico do solo. In: Santos, G. A.; Silva, L. S. S.; Canellas, L. P.; Camargo, F. A. O. (Ed.). (2008), Fundamentos da matéria orgânica do solo: ecossistemas tropicais e subtropicais. Porto Alegre: Grafica Metropole, 87 $-99$.

Cancellier, E. L.; Barros, H. B.; Kischel, E.; Gonzaga, L. A. M.; Brandão, D. R.; Fidelis, R. R. (2011), Eficiência agronômica no uso de nitrogênio mineral por cultivares de arroz de terras altas. Revista Brasileira de Ciências Agrárias. 6, 650-656.

Companhia Nacional de Abastecimento CONAB. (2012) Acompanhamento da safra brasileira: grãos. Disponível em: < http://www.conab.gov.br/OlalaCMS/uploads/arqui vos/12_01_10_10_53_02_boletim_graos_4o_leva ntamento.pdf>. Acesso em: 18 de janeiro de 2012 .

Embrapa - Centro Nacional de Pesquisa de Solos, Sistema brasileiro de classificação de solos. (2006). 2. Ed. Brasília, Embrapa/Dpi. 306p.

Epstein, E.; Bloom, A. J. (2004), Nutrição e Crescimento. In: __ Nutrição mineral de plantas: princípios e perspectivas. $2 . \mathrm{Ed}$. Londrina, Editora Planta, p. 251-284.
Fageria, N. K. (1998), Eficiência de uso de fósforo pelos genótipos de feijão. Revista Brasileira de Engenharia Agrícola e Ambiental, 2, 128- 131.

Fageria, N. D. e Kluthcouski, J. (1980), Metodologia para avaliação de cultivares de arroz e feijão para condições adversas de solo. Brasília: EMBRAPA-CNPAF, 22p.

Fageria, N. K.; Oliveira, I. P. de; Dutra, L. G. (1996), Deficiências nutricionais na cultura do feijoeiro e suas correções. Goiânia: EmbrapaCnpaf, (Documentos), 65, 40p.

Fidelis, R. R, Miranda, G. V.; Faluba, J. S. (2010), Capacidade de combinação de populações de milho tropicais sob estresse de baixo nitrogênio. Bioscience Journal, 26, 358-366.

Kischel, E.; Fidelis, R. R. ; Santos, M. M.; Brandão, D. R.; Cancellier, E. L.; Nascimento, I. R. (2011), Efeito do nitrogênio em genótipos de arroz cultivados em várzea úmida do Estado do Tocantins. Revista Ceres, 58, 84-89.

Lemos, L. B.; Oliveira, R. S.; Palomino, E. C.; Silva, T. R. B. (2004), Características agronômicas e tecnológicas de genótipos de feijão do grupo comercial Carioca. Pesquisa Agropecuária Brasileira, 39, 319-326.

Mechi, R.; Brazaca, S. G. C.; Arthur, V. (2005), Avaliação química, nutricional e fatores antinutricionais do feijão preto (Phaseolus vulgaris L.) irradiado. Ciência e Tecnologia de Alimentos, 25, 109-114.

Posse, S. C. P.; Riva-Souza, E. M.; Silva, G. M.; Fasolo, L. M.; Silva, M. B.; Rocha, M. A. M. (2010), Informações técnicas para o cultivo do feijoeiro-comum na região central-brasileira: 2009-2011, 245p.

Roselem, C. A. e Marubayashi, O. M. (1994) Seja doutor do seu feijoeiro. Botucatu: Potafos, (Arquivo do Agrônomo), 7, 18p.

Rotili, E. A.; Fidelis, R. R.; Santos, M. M.; Barros, H. B.; Pinto, L. C. (2010), Eficiência do uso e resposta à aplicação de fósforo de cultivares de arroz em solos de terras altas. Bragantia, 69, 705710. 
Salgado, F. H. M. Efeito do nitrogênio na produtividade de grãos e qualidade fisiológica de sementes de genótipos de feijão comum. Dissertação (Mestrado em Produção Vegetal) Universidade Federal do Tocantins, 2011.

Salgado, F. H. M.; Fidelis, R. R.; Carvalho, G. L.; Santos, G. R.; Cancellier, E. L.; Silva, G. F. (2011), Comportamento de genótipos de feijão, no período da entressafra, no sul do estado de Tocantins. Bioscience Journal, 27, 52-58.

Sant'ana, E. V. P.; Santos, A. B.; Silveira, P. M. (2010), Adubação nitrogenada na produtividade, leitura spad e teor de nitrogênio em folhas de feijoeiro. Pesquisa Agropecuária Tropical, 40, 491-496.
Santos, A. B, Fageria, N. K, Silva, O. F.; Melo, M. L. B. (2003), Resposta do feijoeiro ao manejo de nitrogênio em várzeas tropicais. Pesquisa Agropecuária Brasileira, 38,1265-1271.

Santos, A. B.; Fageria, N. K. (2007), Manejo do nitrogênio para eficiência de uso por cultivares de feijoeiro em várzea tropical. Pesquisa Agropecuária Brasileira, 42, 1237-1248.

Santos, J. B.; Gavilannes, M. L. (2006), Botânica. In: Vieira, C.; Paula Júnior, T. J.; Borém, A. (Ed.). Feijão. 2. Ed. Viçosa: Universidade Federal de Viçosa, p. 41-65. 